Also available at http://amc.imfm.si

ISSN 1855-3966 (printed edn.), ISSN 1855-3974 (electronic edn.)

ARS MATHEMATICA CONTEMPORANEA 2 (2009) 137-155

\title{
Strongly regular edge-transitive graphs
}

\author{
Joy Morris * \\ Department of Mathematics and Computer Science, University of Lethbridge \\ Lethbridge, AB, TIK 3M4, Canada \\ Cheryl E. Praeger ${ }^{\dagger}$ \\ School of Mathematics and Statistics, University of Western Australia \\ 35 Stirling Highway, Crawley 6009, Western Australia, Australia \\ Pablo Spiga \\ Dipartimento di Matematica Pura ed Applicata, University of Padova \\ Via Trieste 63, 35121 Padova, Italy
}

Received 1 July 2009, accepted 1 September 2009, published online 25 September 2009

\begin{abstract}
In this paper, we examine the structure of vertex- and edge-transitive strongly regular graphs, using normal quotient reduction. We show that the irreducible graphs in this family have quasiprimitive automorphism groups, and prove (using the Classification of Finite Simple Groups) that no graph in this family has a holomorphic simple automorphism group. We also find some constraints on the parameters of the graphs in this family that reduce to complete graphs.
\end{abstract}

Keywords: Strongly regular graphs, vertex-transitive graphs, edge-transitive graphs, normal quotient reduction, automorphism group.

Math. Subj. Class.: 05C25

\section{Introduction}

There has recently been considerable success in using normal quotient reduction to analyse the structure of several families of edge-transitive graphs, including distance-transitive graphs [21], graphs that are $s$-arc-transitive [18, 19], and graphs that are locally $s$-arctransitive [7, 8]. In the first two classes the graphs are also vertex-transitive. This paper

\footnotetext{
${ }^{*}$ Corresponding author.

${ }^{\dagger}$ This author acknowledges support of a Federation Fellowship of the Australian Research Council.

E-mail addresses: joy@cs.uleth.ca (Joy Morris), praeger@maths.uwa.edu.au (Cheryl E. Praeger), spiga@math.unipd.it (Pablo Spiga)
} 
initiates a study of strongly regular graphs that are vertex- and edge-transitive, using normal quotient analysis.

Vertex- and edge-transitive strongly regular graphs were first studied by D. G. Higman in his ground-breaking work on rank 3 groups and graphs [10, 11, 12]. They formed a critical special case that led to his theory of coherent configurations [13]. Some years later Neumaier [17] proved some significant structural results about such graphs when their eigenvalues satisfy a particular inequality. The structures of other families of vertex-transitive strongly regular graphs have been studied by a variety of authors, cf. [1, 2, 14, 15, 16].

A strongly regular graph $(\mathrm{srg})$ with parameters $(n, k, \lambda, \mu)$ is a regular graph with $n$ vertices, valency $k$, such that each pair of adjacent vertices lies in $\lambda$ triangles, and each pair of non-adjacent vertices (if such exist) is joined by $\mu$ paths of length 2 . In particular we regard the complete graph $K_{n}$ as a (somewhat degenerate) strongly regular graph with parameters $(n, n-1, n-2,0)$. To simplify the language in this paper, we use the following terminology throughout.

Definition 1.1. We refer to a vertex- and edge-transitive strongly regular graph as a ve-srg.

Requiring strongly regular graphs to be vertex- and edge-transitive may seem a very stringent condition. However, several important and well-known families of graphs are contained within the class of ve-srgs, making them clearly worthy of study. Notably, Paley graphs, Kneser graphs whose vertices correspond to the 2-element subsets of a set of elements, and more generally, rank 3 graphs are all ve-srgs. Some infinite families of ve-srgs that are not rank 3 can be found in [3].

It is worth noting that the condition of vertex-transitivity in a ve-srg is implied by the rest of the structure.

Lemma 1.2 ([17]). A connected edge-transitive strongly regular graph, is also vertextransitive.

Despite this, the action of a particular group may or may not be transitive on the vertices of the graph, even if it is transitive on the edges. We therefore specify vertex-transitive actions where they are used throughout this paper.

We begin our study by showing that normal quotient reduction (as defined in Section 2 ) applies properly to ve-srgs. That is, we show that each normal quotient of a ve-srg $\Gamma$ is itself a ve-srg. To ensure that the reduction works properly, we do not require that the group $N$ used in the reduction be normal in the full automorphism group of the graph, but we do require that it be nontrivial, intransitive, and normal in a subgroup of $\operatorname{Aut}(\Gamma)$ that is both vertex- and edge-transitive. For this reason, a graph that is irreducible must either be complete, or have the property that every vertex- and edge-transitive subgroup of its automorphism group is quasiprimitive (see Definition 2.6).

This reduction sets up our strategy for determining the structure of ve-srgs: we aim to

1. show that every connected ve-srg can be reduced to an irreducible ve-srg by taking a succession of normal quotients;

2. characterise and as far as possible determine the irreducible ve-srgs: graphs that cannot be further reduced using normal quotient reduction;

3. use what we learn about the irreducible ve-srgs, and the normal quotient structure, to study the structure of arbitrary ve-srgs. 
In Section 2 we discuss normal quotient reduction and in particular we establish (1). In the final three sections of this paper we begin with some minor results on (3), and then achieve some more significant progress on (2).

In Section 3, we consider the structure of ve-srgs that reduce to the trivial case of the complete graph under normal quotient reduction. We completely characterise the structure of ve-srgs that reduce to either $K_{2}$ or $K_{3}$, and find constraints on the parameters of ve-srgs that reduce to one of the larger complete graphs.

In Section 4, we study the family of cartesian products $K_{b} \square K_{b}$. We show that these graphs are ve-srgs, and that graphs in this intriguing family reduce to the complete graph $K_{b}$ if and only if $b$ is a prime power, and are otherwise irreducible.

In Section 5, we show that of the 8 families of quasiprimitive groups, one (holomorphic simple groups) can never arise as the automorphism group of a ve-srg (see Corollary 5.10). This result uses the Classification of Finite Simple Groups.

While the word "connected" appears in many of the results, it is almost unnecessary; a disconnected strongly regular graph is a disjoint union of cliques of some fixed order, whose structure we completely understand.

\section{Normal quotient reduction}

Graph Notation: A graph $\Gamma$ consists of a set $V(\Gamma)$ of vertices and a subset $E(\Gamma)$ of unordered pairs of vertices called edges. An automorphism $g$ of $\Gamma$ is a permutation of $V(\Gamma)$ that leaves $E(\Gamma)$ invariant. We denote the image of a vertex $x$ under $g$ by $g(x)$. For a subgroup $H$ of the automorphism group $\operatorname{Aut}(\Gamma)$ of $\Gamma$, we denote the orbit of $x$ under $H$ by ${ }^{H} x=\{h(x) \mid h \in H\}$ and we say that $\Gamma$ is $H$-vertex-transitive or $H$-edge-transitive if $H$ is transitive on $V(\Gamma)$ or $E(\Gamma)$ respectively. We extend this usage to possibly unfaithful actions: for example, if $H$ acts as a vertex-transitive group of automorphisms of $\Gamma$ with kernel $N$, then we will often say that $\Gamma$ is $H$-vertex-transitive, rather than $(H / N)$-vertextransitive.

In this section, we consider the possibility that $\Gamma$, a ve-srg, has a normal quotient. We look at a vertex-transitive subgroup $G \leq \operatorname{Aut}(\Gamma)$, and suppose that there exists some nontrivial normal subgroup $N$ of $G$ that is intransitive in its action on the vertices of $\Gamma$. In some cases, we may also require that $G$ be transitive on the edges of $\Gamma$.

Definition 2.1. Let $\Gamma$ be a graph and $G$ a vertex-transitive subgroup of $\operatorname{Aut}(\Gamma)$. Suppose that there is some group $N$ such that $1 \neq N \triangleleft G$, and $N$ is intransitive in its action on $V(\Gamma)$.

The quotient graph, $\Gamma_{N}$, is the graph whose vertices are the orbits of $N$, with an edge between two distinct vertices ${ }^{N} x$ and ${ }^{N} y$ in $\Gamma_{N}$, if and only if there is an edge of $\Gamma$ between $x^{\prime}$ and $y^{\prime}$, for some $x^{\prime} \in{ }^{N} x$ and some $y^{\prime} \in{ }^{N} y$.

Sometimes, the original graph will have a nice covering structure with respect to the quotient graph.

Definition 2.2. As before, let $\Gamma$ be a graph, $G \leq \operatorname{Aut}(\Gamma)$ a vertex-transitive subgroup of the automorphism group, and $N$ an intransitive normal subgroup of $G$. Suppose that for each pair $\left\{B, B^{\prime}\right\}$ of adjacent $N$-orbits, each vertex in $B$ is adjacent to exactly $\ell$ vertices in $B^{\prime}$ (so $\ell$ is a constant that does not depend on the choice of $\left\{B, B^{\prime}\right\}$ ). Then we say that $\Gamma$ is an $\ell$-multicover of $\Gamma_{N}$.

Notice that if $\Gamma$ is an $\ell$-multicover of $\Gamma_{N}$, then $\ell$ must divide the valency of $\Gamma$. We now make some observations about the structure of the quotient graph. 
Lemma 2.3. Let $\Gamma$ be a connected ve-srg, with $G \leq \operatorname{Aut}(\Gamma)$ acting transitively on the vertices. Let $1 \neq N \triangleleft G$, for some intransitive $N$, and let $\Gamma_{N}$ be the corresponding quotient graph. Then

1. $\Gamma_{N}$ is connected,

2. $\Gamma_{N}$ is vertex-transitive, and

3. $\Gamma_{N}$ has diameter at most the diameter of $\Gamma$.

Moreover, if $G$ is edge-transitive, then

4. $\Gamma_{N}$ is G-edge-transitive,

5. all edges of $\Gamma$ join vertices in distinct $N$-orbits, and

6. $\Gamma$ is an $\ell$-multicover of $\Gamma_{N}$, for some divisor $\ell$ of the valency of $\Gamma$.

Proof. As (1), (2), (3), and (4) are well-known and their proofs are straightforward, we omit them here.

Property (5) follows immediately from (1) and (4).

(6) Let $\left\{{ }^{N} x,{ }^{N} x^{\prime}\right\}$ be an arbitrary edge of $\Gamma_{N}$, and suppose that there are edges from $x$ to exactly $\ell$ vertices in ${ }^{N} x^{\prime}$. Since the action of $N$ is transitive on ${ }^{N} x$ and fixes every $N$-orbit setwise, it follows that every vertex in ${ }^{N} x$ must have edges to exactly $\ell$ vertices in ${ }^{N} x^{\prime}$. So there are $\left.\ell\right|^{N} x \mid$ edges between the two $N$-orbits. Since $G$ acts transitively on the vertices and $N \triangleleft G,\left|{ }^{N} x\right|=\left|{ }^{N} x^{\prime}\right|$, so considering the action of $N$ on ${ }^{N} x^{\prime}$ shows that every vertex in ${ }^{N} x^{\prime}$ has edges to exactly $\ell$ vertices in ${ }^{N} x$. Now if $\left\{{ }^{N} y,{ }^{N} y^{\prime}\right\}$ is another edge of $\Gamma_{N}$, the fact that $\Gamma_{N}$ is $G$-edge-transitive forces every vertex in ${ }^{N} y$ to be adjacent to exactly $\ell$ vertices in ${ }^{N} y^{\prime}$.

We also deduce that the quotient graph is strongly regular, if the original graph is.

Lemma 2.4. Let $\Gamma$ be a connected ve-srg, with $G \leq \operatorname{Aut}(\Gamma)$ transitive on the vertices and the edges. Let $1 \neq N \triangleleft G$, for some intransitive $N$, and let $\Gamma_{N}$ be the corresponding quotient graph. If $\Gamma$ is complete, then no such quotient is possible; otherwise, $\Gamma_{N}$ is a connected ve-srg.

Proof. We use $(n, k, \lambda, \mu)$ to denote the parameters of $\Gamma$.

That $\Gamma_{N}$ is connected and vertex- and edge-transitive follows from Lemma 2.3(1,2 and 4).

If $\Gamma$ is complete, then any edge-transitive group $G$ is 2 -homogeneous on the vertices of $\Gamma$ (that is, transitive on the unordered 2-sets). All transitive 2-homogeneous groups are primitive (see [5], page 35), so all of their nontrivial normal subgroups are transitive. Thus when $\Gamma$ is complete, there is no nontrivial vertex-intransitive normal subgroup $N$ in $G$. We may therefore assume that $\Gamma$ has diameter 2, as strongly regular graphs have diameter at most 2 .

If $\Gamma_{N}$ is complete, then it is strongly regular, and we are done. So we may also assume that $\Gamma_{N}$ has diameter 2 (by Lemma 2.3(3), its diameter is at most 2).

Since $\Gamma_{N}$ is edge-transitive, each of its edges must lie in a constant number of triangles. This establishes the parameter $\lambda^{\prime}$ of $\Gamma_{N}$.

Since $\Gamma_{N}$ is vertex-transitive, it is clear that $\Gamma_{N}$ is regular, of degree $k^{\prime}$ (say).

Now we wish to show that the number of 2-paths between nonadjacent vertices of $\Gamma_{N}$ does not depend on the choice of the nonadjacent vertices. Let ${ }^{N} x$ and ${ }^{N} y$ be nonadjacent 
vertices of $\Gamma_{N}$. We count the number of 2-paths in $\Gamma$ between the sets ${ }^{N} x$ and ${ }^{N} y$, in two different ways.

First we set up some notation for our counting. Let $b$ denote the number of vertices of $\Gamma$ in each orbit of $N$. By Lemma 2.3(6), $\Gamma$ is an $\ell$-multicover of $\Gamma_{N}$ for some divisor $\ell$ of the valency of $\Gamma$.

Now, the number of 2-paths in $\Gamma$ between ${ }^{N} x$ and ${ }^{N} y$ can be counted as $b$ choices for a vertex $x_{1} \in{ }^{N} x$ to start the path, times $b$ choices for a nonadjacent vertex $y_{1} \in{ }^{N} y$ to end the path, times $\mu$ 2-paths between $x_{1}$ and $y_{1}$, since $\Gamma$ is strongly regular.

Alternatively, the number of 2-paths in $\Gamma$ between ${ }^{N} x$ and ${ }^{N} y$ can be counted as $b$ choices for a vertex $x_{1} \in{ }^{N} x$ to start the path, times $\mu_{N_{x}{ }^{N}{ }_{y}}^{\prime}$ choices for a set ${ }^{N} u$ that is mutually adjacent to ${ }^{N} x$ and ${ }^{N} y$, times $\ell$ choices for a vertex $u_{1} \in{ }^{N} u$ that is adjacent to $x_{1}$, times $\ell$ choices for a vertex $y_{1} \in{ }^{N} y$ that is adjacent to $u_{1}$.

These two ways of counting the same thing, show us that

$$
b^{2} \mu=b \mu_{N}^{\prime} x,^{N} \ell^{2} .
$$

Since none of $b, \mu$ and $\ell$ depends on the choices of ${ }^{N} x$ and ${ }^{N} y$, we have that $\mu_{N,{ }^{N} y}^{\prime}=$ $b \mu / \ell^{2}$ must not depend on the choices of ${ }^{N} x$ and ${ }^{N} y$, either. Thus the number of 2-paths between nonadjacent vertices of $\Gamma_{N}$ does not depend on the choice of the nonadjacent vertices.

We have shown that a normal quotient of a connected ve-srg, is itself a connected vesrg.

Corollary 2.5. Let $\Gamma$ (not a complete graph), $G, N$, and $\Gamma_{N}$ be as in Lemma 2.4, and let the parameters of $\Gamma$ be $(n, k, \lambda, \mu)$. As in the proof of Lemma 2.4, let b be the length of the orbits of $N$, and let $\ell$ be such that $\Gamma$ is an $\ell$-multicover of $\Gamma_{N}$.

Then the quotient graph $\Gamma_{N}$ has parameters $\left(n^{\prime}, k^{\prime}, \lambda^{\prime}, \mu^{\prime}\right)$, with

1. $n^{\prime}=n / b$;

2. $k^{\prime}=k / \ell$;

3. $\lambda^{\prime}=[\ell \lambda+(b-\ell) \mu] / \ell^{2}$; and

4. $\mu^{\prime}=b \mu / \ell^{2}$ unless $\Gamma_{N}$ is complete.

Proof. The values of $n^{\prime}$ and $k^{\prime}$ come directly from the definitions of quotient graphs and $\ell$-multicovers. The value of $\mu^{\prime}$ comes from the proof of Lemma 2.4, and a very similar counting argument (double-counting paths of length 2 in $\Gamma$ between adjacent $N$-orbits) produces the given value for $\lambda^{\prime}$.

Notice that if $\Gamma$ is a graph that cannot be further reduced using this normal quotient reduction then either $\Gamma$ is complete, or $\Gamma$ has no group $G$ of automorphisms that is transitive on both the vertices and the edges and that has a subgroup $N$ such that $1 \neq N \triangleleft G$ is vertexintransitive. The following definition is therefore very important.

Definition 2.6. A transitive permutation group is said to be quasiprimitive if every nontrivial normal subgroup is transitive. 
Thus, the "irreducible" connected ve-srgs are the complete graphs, together with the graphs for which every vertex- and edge-transitive group of automorphisms is quasiprimitive.

It might be thought that the requirement of edge-transitivity is artificial; it certainly creates some noteworthy awkwardness in at least two ways. Firstly, the complement of a connected ve-srg will be strongly regular, vertex-transitive and often connected, but edgetransitivity is unlikely. Secondly, we will see later with the example of the cartesian product $K_{b} \square K_{b}$ that there may be ve-srgs with normal quotients that are also ve-srgs, but even though both the graph and the quotient are edge-transitive, the reduction can only be made by taking a normal subgroup of a vertex-transitive subgroup of the automorphism group, not by taking a normal subgroup of a vertex- and edge-transitive subgroup of the automorphism group. However, it seems to be very difficult to determine much information about these graphs if we drop the requirement that $G$ be edge-transitive.

We will consider first the degenerate case of graphs for which some normal quotient is a complete graph, and therefore irreducible.

\section{Complete graphs as normal quotients}

In this section, we consider the degenerate case in which some normal quotient of a ve-srg is a complete graph. We analyse the structure of the original graph in this case.

We will be using the same hypotheses repeatedly in this section, so to shorten the statements of the results, we state these hypotheses here.

Hypothesis 3.1. Let $\Gamma$ be a connected ve-srg with parameters $(n, k, \lambda, \mu)$, and $G \leq$ Aut $(\Gamma)$ acting transitively on the vertices and on the edges. Let $1 \neq N \triangleleft G$, for some intransitive $N$, and let $\Gamma_{N}$ be the corresponding quotient graph. Furthermore, let b denote the number of vertices in each orbit of $N$; $m$ the valency of $\Gamma_{N}$; and $\ell=k / m$ the number of edges from $x$ to ${ }^{N} y$ whenever ${ }^{N} x$ and ${ }^{N} y$ are adjacent in $\Gamma_{N}$.

Lemma 3.2. Under Hypotheses 3.1, if $\Gamma_{N}=K_{2}$, then $\Gamma$ is a complete bipartite graph.

Proof. Let ${ }^{N} x$ and ${ }^{N} y$ be the two vertices of $\Gamma_{N}$. By Lemma 2.3(5), every edge of $\Gamma$ has one endvertex in ${ }^{N} x$ and the other in ${ }^{N} y$, so $\Gamma$ is bipartite.

Since $\Gamma$ is strongly regular, it has diameter at most 2 . Since two vertices in opposite sets of the bipartition must be at an odd distance, any two such vertices must be at distance 1. Thus, $\Gamma$ is a complete bipartite graph.

This result does not generalise, but we can draw some conclusions about the parameters of ve-srgs with a complete normal quotient.

Proposition 3.3. Under Hypotheses 3.1, if $\Gamma_{N}=K_{m+1}$ for some $m \geq 1$, then

1. $(b-1) \mu=\ell m(\ell-1)$ and in particular $\ell \geq 2$,

2. $(b-\ell) \mu=\ell(\ell m-\ell-\lambda)$,

3. $\mu(\ell-1)=\ell(\ell+\lambda-m)$, and

4. $\ell \mid \mu$,

Furthermore, if $\Gamma$ is not the complete multipartite graph $K_{(m+1)[b]}$, then

5. $\mu \leq(m-1) \ell$. 
Proof. By Lemma 3.2, the result holds for $m=1$. So in what follows, we may assume $m \geq 2$. By Lemma 2.3(5), every edge of $\Gamma$ lies between orbits, so $\Gamma$ is multipartite.

We count the number of 2-paths that start at a fixed vertex $v$, and end in ${ }^{N} v$, in two ways. First, with $v$ fixed, there are $b-1$ ways to choose a vertex $v^{\prime} \in{ }^{N} v$, to be the terminal vertex of the 2-path. Since $v$ and $v^{\prime}$ are in the same $N$-orbit, they are nonadjacent by Lemma 2.3(5), so there are $\mu$ different 2-paths from $v$ to $v^{\prime}$ to choose amongst. This makes $(b-1) \mu$ 2-paths in all. Alternatively, again with $v$ fixed, we can choose an adjacent vertex $w$ in $k=\ell m$ ways. For each such choice, we can choose any of the $\ell-1$ vertices of ${ }^{N} v$ that are not $v$ but that are adjacent to $w$, to complete the 2-path. Thus, we conclude that

$$
(b-1) \mu=\ell m(\ell-1) \text {. }
$$

This is the first of our desired conclusions. Furthermore, since $N \neq 1$, we have $b>1$, and by Lemma 2.4, $\Gamma$ is not complete, so $\Gamma$ has diameter 2, meaning $\mu \neq 0$. Thus Equation $3 \mathrm{~A}$ forces $\ell \geq 2$.

With a fixed starting vertex $v$, we count the number of 2-paths that start at $v$ and end at some vertex $u \notin^{N} v$ for which $v$ and $u$ are not adjacent (if there is no such vertex $u$, our count will produce 0 ). Fixing $v$, there are $m$ orbits of $N$ that do not contain $v$, and since $\Gamma_{N}=K_{m+1}$, each of these contains $b-\ell$ vertices that are not adjacent to $v$ that serve as choices for the terminal vertex $u$ of our 2-path. For each choice of $u$, there are $\mu$ 2-paths from $v$ to $u$ from which we may choose. Thus, the number of 2-paths is $m(b-\ell) \mu$. Alternatively, again with $v$ fixed, we can choose an adjacent vertex $w$ in $k=\ell m$ ways. For each such choice, there are $\ell m-\ell$ vertices that are adjacent to $w$ but not in ${ }^{N} v$; however, $\lambda$ of these vertices are actually adjacent to $v$ also (recall that none of the vertices in ${ }^{N} v$ are adjacent to $v$ by Lemma 2.3(5)), so in total $\ell m(\ell m-\ell-\lambda)$ is the number of 2-paths starting at $v$ and terminating in some $u \notin{ }^{N} v$ that is not adjacent to $v$. We conclude that

$$
m(b-\ell) \mu=\ell m(\ell m-\ell-\lambda),
$$

and dividing through by $m$,

$$
(b-\ell) \mu=\ell(\ell m-\ell-\lambda),
$$

our second conclusion.

Now we take Equation 3A and subtract Equation 3B, to obtain

$$
\mu(\ell-1)=\ell(\ell+\lambda-m),
$$

our third conclusion. Furthermore, since $\ell$ and $\ell-1$ are coprime, this forces $\ell \mid \mu$, our fourth conclusion.

Finally, suppose that $\Gamma$ is not $K_{(m+1)[b]}$, and fix two nonadjacent vertices $v, u$ in distinct blocks (such vertices exist since $\Gamma$ is not complete multipartite). The first vertex $v$ has $(m-1) \ell$ neighbours in blocks other than ${ }^{N} u$. Since any 2-path between the vertices must go through a third block (by Lemma 2.3(5)), the number $\mu$ of 2-paths between $v$ and $u$ cannot be more than $(m-1) \ell$. This is the last of our desired conclusions.

In the case where $\Gamma$ is not complete multipartite, then, we have (among other things) that $\ell \mid \mu$, but $\mu \leq(m-1) \ell$. It would seem to be interesting to determine how these relationships between $\mu$ and $\ell$ affect the graph $\Gamma$, and for which values of $\mu$ relative to $\ell$ there in fact are strongly regular, connected, edge-transitive graphs. We begin by considering one extreme of the relation: the case where $\mu=(m-1) \ell$. 
Proposition 3.4. Under Hypotheses 3.1, if $\Gamma_{N}=K_{m+1}$ for some $m \geq 2$ and $\mu=(m-$ 1) $\ell$, then $\Gamma \cong K_{b[b]}-b K_{b}$, the complete b-partite graph with $b$ vertices in each part, with the edges of $b$ vertex-disjoint copies of $K_{b}$ deleted, and $b=\ell+1=m+1$.

Proof. The graph $\Gamma$ cannot be $K_{(m+1)[b]}$ since for that graph $\mu=m \ell$. Therefore Proposition 3.3(1) gives $(b-1) \mu=\ell m(\ell-1)$, so $(b-1)(m-1)=m(\ell-1)$. Thus $b-1=\left(1+\frac{1}{m-1}\right)(\ell-1) \leq 2(\ell-1)$, so $b \leq 2 \ell-1$.

Fix a vertex $v$. If $b>\ell+1$, then there are at least two vertices $u$ and $w$ in some other block, both of which are nonadjacent to $v$. Since $\mu=(m-1) \ell$, both $u$ and $w$ must have exactly the same neighbours as $v$ in the remaining $m-1$ blocks, to create $\mu$ 2-paths between $v$ and $u$, and $\mu$ 2-paths between $v$ and $w$. Since these $\mu$ mutual neighbours also create $\mu$ 2-paths between $u$ and $w$, it must be that $u$ and $w$ have no mutual neighbours in the block containing $v$. But since each has $\ell$ neighbours in the block containing $v$, and there are a total of $b \leq 2 \ell-1$ vertices in that block, the pigeon-hole principle forces $u$ and $w$ to have at least one mutual neighbour in the block containing $v$, a contradiction. We can thus conclude that $b \leq \ell+1$. In fact, since $b \geq \ell$ by definition of $\ell$, and $b=\ell$ would give a complete multipartite graph, we can conclude that $b=\ell+1$.

By Proposition 3.3(1) with $b=\ell+1$, we obtain $\mu=m(\ell-1)$. By assumption, then, $(m-1) \ell=m(\ell-1)$, which forces $m=\ell=b-1$. We also have $\mu=\ell(\ell-1)$ and $\lambda=(\ell-1)^{2}$ (from Proposition 3.3, parts (1) and (3)). These parameters force the structure that we have claimed, as we will show in the next paragraph.

Towards a contradiction, suppose that for some vertex $v$, the set of non-neighbours of $v$ that are not in ${ }^{N} v$ is not an independent set of vertices. Then there are two non-neighbours of $v, u$ and $w$ (say), that are adjacent, and $u, v$ and $w$ are all in different blocks. Now, since each of $u$ and $w$ has $\ell=b-1$ neighbours in each of the blocks that do not contain $u$ or $w$, they must have either $\ell$ or $\ell-1$ common neighbours in each of these blocks. Since there are $\ell-1$ such blocks, and they have a total of $(\ell-1)^{2}$ mutual neighbours, they must have exactly $\ell-1$ mutual neighbours in each of these blocks. But since both $u$ and $w$ are non-neighbours of $v$, they have $\ell$ mutual neighbours in the block ${ }^{N} v$, a contradiction.

It is not hard to verify that the graphs $K_{b[b]}-b K_{b}$ form an infinite family of connected ve-srgs that are not complete multipartite, and that have complete normal quotients.

Interestingly, a consequence of the preceding result is that there is only one ve-srg that is not complete tripartite and has $K_{3}$ as a normal quotient.

Corollary 3.5. Under Hypotheses 3.1, if $\Gamma_{N}=K_{3}$, then either $\Gamma \cong K_{3[b]}$, or $b=3$ and $\Gamma \cong K_{3[3]}-3 K_{3}$.

Proof. Here we have $m=2$. We assume that $\Gamma$ is not $K_{3[b]}$.

Since $m=2$, Proposition 3.3(4) and (5) give $\ell \mid \mu$ (so $\ell \leq \mu$ ), and $\mu \leq \ell$. Thus $\mu=(m-1) \ell=\ell$. Now Proposition 3.4 completes the proof.

At the other extreme lies the possibility that $\mu=\ell$. Here there is another infinite family of connected ve-srgs: the graphs $K_{b} \square K_{b}$. As there is quite a bit to say about these graphs, we will discuss them in a separate section, shortly.

Meanwhile, putting additional standard facts about strongly regular graphs together with the results of Proposition 3.3, we can produce a bit more information about the possible parameters of other ve-srgs $\Gamma$ that reduce to complete graphs. By the eigenvalues of $\Gamma$ we mean the eigenvalues of its adjacency matrix (see [9]). 
Proposition 3.6. Under Hypotheses 3.1, if $\Gamma_{N}=K_{m+1}$, then either $\Gamma$ is a complete multipartite graph, or the eigenvalues of $\Gamma$ are

$$
k, \theta=m-r, \text { and } \tau=-\ell,
$$

where $\ell=k / m$ and $r=\mu / \ell$. Furthermore, the multiplicities of the eigenvalues $\theta$ and $\tau$ are

$$
\begin{aligned}
m_{\theta} & =\frac{m \ell(m+1)(\ell-1)}{r(m-r+\ell)} \\
m_{\tau} & =\frac{m(m-r+1)(m \ell+r-m)}{r(m-r+\ell)}
\end{aligned}
$$

so these values must be integers.

The parameters for $\Gamma$ are

$$
((m+1)(m \ell-m+r) / r, m \ell,(\ell-1) r+m-\ell, r \ell) .
$$

Proof. We assume that $\Gamma$ is not complete multipartite, and deduce the given formulae for the eigenvalues, their multiplicities, and the parameters of $\Gamma$. Standard results (cf. [9], p. 220) on strongly regular graphs give the formulas

$$
\theta=(\lambda-\mu+\sqrt{\Delta}) / 2 \text { and } \tau=(\lambda-\mu-\sqrt{\Delta}) / 2,
$$

where $\Delta=(\lambda-\mu)^{2}+4(k-\mu)$. Solving for $\lambda$ in Proposition 3.3(3) gives $\lambda=(\ell-1) r+$ $m-\ell$ (which is the value given above for the third parameter of $\Gamma$ ). Substituting this into the formula for $\Delta$ gives $\Delta=(m+\ell-r)^{2}$. The values given for $\theta$ and $\tau$ are an immediate consequence of combining these results.

Similarly, standard results on strongly regular graphs give

$$
m_{\theta}=\frac{(n-1) \tau+k}{\tau-\theta} \text { and } m_{\tau}=\frac{(n-1) \theta+k}{\theta-\tau} .
$$

Solving for $b$ in Proposition 3.3(1) gives $b=(m \ell-m+r) / r$, and we know $n=(m+1) b$. This yields the given value for $n$ in the parameters of $\Gamma$, completing the calculations of the parameters. Using this value for $n$ in the formulas for $m_{\theta}$ and $m_{\tau}$ completes the result.

While we have determined some information about ve-srgs that reduce to the complete graph, and have determined some special cases, there is much still to be explored here.

\section{The family $K_{b} \square K_{b}$}

In this section, we consider the infinite family of graphs consisting of cartesian products of two copies of a complete graph. These graphs are also known as Hamming graphs with diameter 2 , or as $b \times b$-grid graphs. First we show that these graphs are all connected ve-srgs. Then we show that while all have $K_{b}$ as a normal quotient relative to a vertextransitive subgroup of automorphisms, the family is divided into two infinite subfamilies. The graphs $\Gamma$ in the first subfamily have an edge-transitive group $G$ of automorphisms and a nontrivial normal subgroup $N$ of $G$, such that $\Gamma_{N}=K_{b}$. In the other subfamily, every edge-transitive group of automorphisms is quasiprimitive, so graphs in the second subfamily are irreducible.

We define $\Gamma=K_{b} \square K_{b}$ as the graph with vertices the pairs $(i, j)$ with $i, j \in \mathbb{Z}_{b}$, such that $(i, j)$ and $\left(i^{\prime}, j^{\prime}\right)$ form an edge if and only if $i=i^{\prime}$ or $j=j^{\prime}$ (but not both). 
Proposition 4.1. Let $\Gamma=K_{b} \square K_{b}$. Then $\Gamma$ is vertex- and edge-transitive and strongly regular with parameters $\left(b^{2}, 2 b-2, b-2,2\right)$.

Furthermore, $\Gamma$ is a b-partite graph with parts of cardinality $b$. In fact, $K_{b}$ is a normal quotient of $K_{b} \square K_{b}$ relative to a vertex-transitive subgroup of $\operatorname{Aut}(\Gamma)$, and $\Gamma$ is a 2-multicover of this quotient.

Proof. It is well-known and easy to show that $\Gamma$ is vertex- and edge-transitive. The number of vertices is $b^{2}$ and the valency is $2 b-2$. Every edge is in a unique $K_{b}$, either formed by the $b$ vertices with the same first coordinate, or by the $b$ vertices with the same second coordinate. No edge is in any other triangles. This establishes that $\lambda=b-2$.

If $(i, j)$ and $\left(i^{\prime}, j^{\prime}\right)$ are not adjacent, then $j \neq j^{\prime}$ and $i \neq i^{\prime}$. The only vertices adjacent to both of these vertices, are $\left(i, j^{\prime}\right)$ and $\left(i^{\prime}, j\right)$. This establishes that $\mu=2$ and that $\Gamma$ is indeed strongly regular.

Choose the diagonal sets $\left\{\left\{(i, i+j): i \in \mathbb{Z}_{b}\right\}: j \in \mathbb{Z}_{b}\right\}$ as the partition sets. Then it is clear that $\Gamma$ is $b$-partite with parts of cardinality $b$, and each vertex is joined to exactly two vertices in each partition set apart from the one containing it.

Let $G$ be $\mathbb{Z}_{b} \times \mathbb{Z}_{b}$ in its action by left multiplication on the vertices of $\Gamma$. Then $G$ is a subgroup of $\operatorname{Aut}(\Gamma)$ that acts transitively on the vertices. Furthermore, as $G$ is abelian, the subgroup $N=\left\{n_{x}: x \in \mathbb{Z}_{b}\right\}$, where $n_{x}(i, j)=(i+x, j+x)$, is normal in $G$, and the $N$-orbits are the partition sets mentioned above. Thus $\Gamma_{N} \cong K_{b}$ and $\Gamma$ is a 2 -multicover of $\Gamma_{N}$.

Remark 4.2. We can also obtain $K_{b}$ as a normal quotient by taking $G$ to be $S_{b} \times S_{b}$, which again is vertex-transitive but not edge-transitive, and $N=S_{b} \times 1$. However in this case $\Gamma$ is not a multicover of $\Gamma_{N}$, since the partition sets this time contain edges of $\Gamma$.

Unfortunately, an unexpected artifact of our requirement that the subgroup $G$ of $\operatorname{Aut}(\Gamma)$ used in these results must be transitive on the edges as well as on the vertices, is that some, but not all, of the graphs in this family actually reduce to the complete graph using our normal quotient reduction scheme, even though both the original graphs in this family and the quotients are edge-transitive.

Proposition 4.3. Let $\Gamma$ be the cartesian product $K_{b} \square K_{b}$. If $b$ is a prime power, then there is a vertex-and edge-transitive sugroup $G \leq \operatorname{Aut}(\Gamma)$ with a normal subgroup $N$ such that $\Gamma_{N} \cong K_{b}$.

Proof. Let GF $(b)$ denote the field of order $b$, and label the vertices of $\Gamma$ by pairs $(i, j)$ with $i, j \in \mathrm{GF}(b)$ such that $(i, j)$ and $\left(i^{\prime}, j^{\prime}\right)$ form an edge if and only if $i=i^{\prime}$ or $j=j^{\prime}$ (but not both).

Let $n_{x}(i, j)=(i+x, j+x)$ for every $i, j, x \in \mathrm{GF}(b)$, and let $N=\left\{n_{x}: x \in \mathrm{GF}(b)\right\}$. Then $N \leq \operatorname{Aut}(\Gamma)$. The orbits of $N$ are the $b$ transversals $\{(i+x, x): x \in \operatorname{GF}(b)\}$, one for each value of $i$. Also, as in the proof of Proposition 4.1, $N$ is a normal subgroup of a vertex-transitive subgroup of $\operatorname{Aut}(\Gamma)$, and $\Gamma_{N}=K_{b}$. It remains to be shown that there exists some subgroup $G$ of the normaliser of $N$ in $\operatorname{Aut}(\Gamma)$, such that $G$ is both vertex- and edge-transitive.

Let $g_{r, s, s^{\prime}}(i, j)=\left(r i+s, r j+s^{\prime}\right)$ for every $i, j, s, s^{\prime} \in \mathrm{GF}(b)$, and for every $r \in$ $\mathrm{GF}(b)^{*}$. Let $G^{\prime}=\left\{g_{r, s, s^{\prime}}: s, s^{\prime} \in \mathrm{GF}(b), r \in \mathrm{GF}(b)^{*}\right\}$. Let $\delta(i, j)=(j, i)$ for every $i, j \in \mathrm{GF}(b)$, and let $G=\left\langle G^{\prime}, \delta\right\rangle$. Then it is straightforward to see that $N \triangleleft G$ and $G \leq \operatorname{Aut}(\Gamma)$. 
We claim that $G$ acts transitively on the vertices and edges of $\Gamma$. By varying $s$ and $s^{\prime}$ with $r=1$, it is clear that $G$ acts transitively on the vertices of $\Gamma$. The group generated by the automorphisms $g_{r, 0,0}$ together with $\delta$ fixes the vertex $(0,0)$ and acts transitively on its neighbours, so $G$ does indeed act transitively on the edges of $\Gamma$.

Our next series of results shows that in fact, the graphs $K_{b} \square K_{b}$ reduce to $K_{b}$ only if $b$ is a prime power, and that otherwise, these graphs are irreducible.

Lemma 4.4. Let $\Gamma$ be the cartesian product $K_{b} \square K_{b}$. If $G \leq \operatorname{Aut}(\Gamma)$ acts transitively on the vertices and the edges, then the orbits of any nontrivial intransitive normal subgroup of $G$ must:

\section{1. be independent sets in $K_{b} \square K_{b}$;}

2. have length $b$; and

3. yield a complete normal quotient $\Gamma_{N} \cong K_{b}$.

Proof. Let $N$ be a nontrivial intransitive normal subgroup of $G$. Then the $N$-orbits in $V(\Gamma)$ form blocks of imprimitivity for the action of $G$. Since $G$ is edge-transitive and $\Gamma$ is connected, there cannot be any edges between vertices that lie in the same (nontrivial) block of imprimitivity of $G$. This establishes that since $N$ is intransitive, the orbits of $N$ are independent sets (partial transversals) of $K_{b} \square K_{b}$.

Since $N$ is nontrivial, its orbits must have length greater than 1 . Let $(x, y)$ and $\left(x^{\prime}, y^{\prime}\right)$ be distinct vertices in one orbit of $N$. These two vertices are not adjacent and so $x \neq$ $x^{\prime}, y \neq y^{\prime}$. Since this orbit is an independent set, $\left(x, y^{\prime}\right)$ is in a different $N$-orbit, and these orbits are clearly adjacent in $\Gamma_{N}$. Furthermore, since ${ }^{N}(x, y)$ is independent, $(x, y)$ and $\left(x^{\prime}, y^{\prime}\right)$ are the only vertices of ${ }^{N}(x, y)$ with first entry $x$ or second entry $y^{\prime}$, respectively, and hence they are the only vertices of ${ }^{N}(x, y)$ which are adjacent to $\left(x, y^{\prime}\right)$. Since $\Gamma$ is an $\ell$-multicover of $\Gamma_{N}$, this means that $\ell=2$. Now, if ${ }^{N}(x, y)$ has length less than $b$, then there is some $j \in \mathrm{GF}(b)$ such that no vertex of ${ }^{N}(x, y)$ has second entry $j$. Hence $(x, j)$ is not in ${ }^{N}(x, y)$, and has exactly one neighbour $(x, y)$ in ${ }^{N}(x, y)$, contradicting $\ell=2$. Thus, the orbits ${ }^{N}(x, y)$ must have length at least $b$. As they are independent sets, they have length exactly $b$, and must contain exactly one pair from each row and each column. It follows that the normal quotient $\Gamma_{N}$ is a complete graph $K_{b}$.

Proposition 4.5. Let $\Gamma$ be the cartesian product $K_{b} \square K_{b}$. Let $G \leq \operatorname{Aut}(\Gamma)$ act transitively on the vertices and the edges of $\Gamma$. If $b$ is not a prime power, then $G$ is quasiprimitive.

Proof. The automorphism group of $K_{b} \square K_{b}$ is $S_{b} 2 S_{2}$ (the wreath product of $S_{b}$ and $S_{2}$ ), and is generated by $B=S_{b} \times S_{b}$, together with $\delta$, where $\delta((i, j))=(j, i)$. Notice that $B \triangleleft \operatorname{Aut}(\Gamma)$; in fact, $B$ has index 2 in $\operatorname{Aut}(\Gamma)$. Furthermore, each row and column of $\Gamma$ is a block of imprimitivity for $B$, so for any subgroup of $B$, it is reasonable to talk about the action on the rows and on the columns of $\Gamma$.

Let $G$ be a subgroup of $\operatorname{Aut}(\Gamma)$ that acts transitively on the vertices and edges of $\Gamma$, and let $G^{\prime}=G \cap B$. Since $G$ is edge-transitive, $G$ cannot be contained in $B$. So $G^{\prime}$ has index 2 in $G$. Furthermore, the action of $G^{\prime}$ is 2-homogeneous on the rows and on the columns of $K_{b} \square K_{b}$. 
Let $N \triangleleft G$, with $N$ nontrivial and intransitive, and let $N^{\prime}=N \cap G^{\prime}$. As $N^{\prime}$ is the intersection of two normal subgroups of $G$, we have $N^{\prime} \triangleleft G$. The remainder of the proof breaks down into two cases, depending on whether or not $N^{\prime}=1$.

Case 1. $N^{\prime}=1$. Then $N \cap G^{\prime}=1$, so $|N|=2$ and $G=G^{\prime} N$. Hence $G=G^{\prime} \times N$. Let $n \in N$ with $n \neq 1$. Now $n \in G \backslash B$, so $n=\delta(\sigma, \tau)$, where $(\sigma, \tau) \in B$. Since $|N|=2$ we have

$$
\begin{aligned}
1=n^{2} & =\delta(\sigma, \tau) \delta(\sigma, \tau) \\
& =\delta^{2}(\tau \sigma, \sigma \tau)
\end{aligned}
$$

which forces $\tau=\sigma^{-1}$.

Now

$$
\begin{aligned}
n\left(i,{ }^{\sigma} i\right) & =\delta\left(\sigma, \sigma^{-1}\right)\left(i,{ }^{\sigma} i\right) \\
& =\delta\left({ }^{\sigma} i, i\right) \\
& =\left(i,{ }^{\sigma} i\right)
\end{aligned}
$$

so for every $i,\left(i,{ }^{\sigma} i\right)$ is a fixed point of $n$ and hence a fixed point of $N=\{1, n\}$. But the orbits of $N$ are blocks of the transitive group $G$, so all have the same length, a contradiction.

Case 2. $N^{\prime} \neq 1$. Then $N^{\prime}$ is a nontrivial, intransitive subgroup of $G$. By Lemma 4.4(1 and 2), the orbits of $N^{\prime}$ on the vertices of $\Gamma$ have length $b$ and are independent sets, so consist of one vertex from each row. Therefore, the orbits of $N^{\prime}$ in its action on the rows must have length $b$; that is, $N^{\prime}$ is transitive on the rows. Similarly, $N^{\prime}$ is transitive on the columns.

Suppose that for some $n \in N^{\prime}$, the action of $n$ fixes row $i$ setwise. Then for any column $j, n((i, j))=(i, j)$ since the orbits of $N^{\prime}$ are independent sets (by Lemma 4.4(1)). Thus $n$ fixes every column setwise. Similarly, since $n$ now fixes column $j, n$ fixes every row setwise. This shows that the action of $N^{\prime}$ is faithful and regular on the rows. Then, since $N^{\prime} \triangleleft G^{\prime}$ and $G^{\prime}$ is 2-homogeneous on the rows, it follows that $N^{\prime}$ is elementary abelian, and in particular $b$ is a prime power.

This shows that $G$ cannot have a nontrivial, intransitive normal subgroup if $b$ is not a prime power. By definition of quasiprimitivity, $G$ must be quasiprimitive if $b$ is not a prime power.

Combining the preceding results yields the following information about this family of graphs.

Corollary 4.6. Let $\Gamma$ be the cartesian product $K_{b} \square K_{b}$. There exists a vertex- and edgetransitive group $G \leq \operatorname{Aut}(\Gamma)$ with a nontrivial, vertex-intransitive, normal subgroup $N$ if and only if $b$ is a prime power. Moreover in this case each such subgroup $N$ corresponds to a complete normal quotient $\Gamma_{N} \cong K_{b}$.

Proof. If $b$ is a prime power, the existence of the groups $G$ and $N$ follows from Proposition 4.3, and the fact that each intransitive $N$ corresponds to a complete normal quotient $\Gamma_{N} \cong K_{b}$ follows from Lemma 4.4(3). If $b$ is not a prime power, the quasiprimitivity of $G$ from Proposition 4.5 shows that no such $N$ exists.

Juxtaposing the fact that $K_{b}$ is always a normal quotient of $K_{b} \square K_{b}$ relative to a vertextransitive group $G$, with the fact that $G$ can be edge-transitive if and only if $b$ is a prime 
power, illustrates the complex behaviour of these Cartesian products. Our results show that either these graphs are themselves irreducible, or they reduce to a complete graph $K_{b}$ (which is irreducible) under normal quotient reduction.

\section{Irreducible graphs and holomorphic simple groups}

By our reduction method, if a graph that is not complete is to be irreducible, then every group of automorphisms that acts vertex- and edge-transitively on the graph must be quasiprimitive.

According to Praeger's characterisation of quasiprimitive groups [19], they fall into one of the following families:

1. holomorphic simple groups;

2. affine groups;

3. almost simple groups;

4. simple diagonal action groups with one minimal normal subgroup;

5. compound holomorphic groups;

6. compound diagonal action groups;

7. product action groups; and

8. twisted wreath action groups.

While some of these groups do act as automorphism groups of ve-srgs (such as affine groups acting on the Paley graphs, and certain simple group actions on ve-srgs), we will devote the remainder of this paper to proving that holomorphic simple groups cannot arise as vertex- and edge-transitive automorphism groups of ve-srgs. This result is stated in Corollary 5.10.

We recall the definition of homomorphic simple groups. Let $T$ be a nonabelian simple group and $W=T \rtimes \operatorname{Aut}(T)$. The group $W$ has a natural action on the set $T$, where the subgroup $T$ of $W$ acts by left multiplication and the subgroup $\operatorname{Aut}(T)$ of $W$ acts by automorphisms. With this permutation representation, $W$ is a primitive subgroup of $\operatorname{Sym}(T)$. We note that the stabilizer of the point $1_{T}$ in $W$ is $\operatorname{Aut}(T)$. Any subgroup $G$ of $W$ containing $T \rtimes \operatorname{Inn} T$ is said to be a holomorphic simple group. In this case, the group $G$ is quasiprimitive (it is actually primitive), with exactly two minimal normal subgroups, $T$ and $M=C_{G}(T)$. Furthermore, $T \cong M$ and $T \rtimes \operatorname{Inn} T=T \times M$.

Remark 5.1. If a holomorphic simple group $G$ (as above) has a vertex-transitive action on a graph $\Gamma$, then (by definition of quasiprimitive) $T$ and $M$ act transitively on the vertices of $\Gamma$. In fact, $T$ and $M$ act regularly on the vertices of $\Gamma$ (the subgroups $T$ and $M$ of $G$ are the left and right regular permutation representations of the simple group $T$ ). Thus $\Gamma$ is a Cayley graph on $T$ (or on $M$ ).

This remark shows that if $G$ is a holomorphic simple group acting vertex-transitively on $\Gamma$, we can identify the vertices of $\Gamma$ with the elements of the simple group $T$.

Again, we have a series of hypotheses that we will be using in many of the results in this section, so we collect them here. 
Hypothesis 5.2. Let $\Gamma$ be a connected ve-srg with parameters $(n, k, \lambda, \mu)$, and with $G \leq$ $\operatorname{Aut}(\Gamma)$ acting transitively on the vertices and on the edges. Suppose that $G$ is a holomorphic simple group, with minimal normal subgroups $T$ and $M$. Identify the vertices of $\Gamma$ with the elements of $T$, according to Remark 5.1. Let $H=G_{1_{T}}$, the stabiliser of the identity, and let $S$ be the connection set of the Cayley graph $\Gamma$. Let $y=\operatorname{gcd}(|H: \operatorname{Inn} T|,|T|-1)$.

We need to consider what the connection set of such a Cayley graph might look like. The following result is a special case of Proposition 1 of [20], but as the proof is short we include it here.

Lemma 5.3. Under Hypotheses 5.2, either $S$ is an orbit of $H$, or for every $s \in S$ there is no $h \in H$ such that $h(s)=s^{-1}$. In the latter case, $S$ is the union of two orbits $B$ and $B^{-1}$ of $H$.

Proof. Suppose $s, s^{\prime} \in S$ so that $\left\{1_{T}, s\right\}$ and $\left\{1_{T}, s^{\prime}\right\}$ are edges of $\Gamma$. Since $G$ is edgetransitive, there is either some $h \in H$ such that $h(s)=s^{\prime}$, or some $g \in G$ such that $g$ maps $s$ to $1_{T}$ and $1_{T}$ to $s^{\prime}$. But as $G=H T$, if $g=h t$ with $t \in T$ and $h \in H$, we must have $t=s^{-1}$ and $h$ takes $s^{-1}$ to $s^{\prime}$. Thus, $s^{\prime}$ is in the same $H$-orbit as either $s$ or $s^{-1}$.

Now we can find an upper bound on the order of the centraliser of any element in the connection set.

Lemma 5.4. Under Hypotheses 5.2, we have $\Gamma ¥ K_{n}, y \geq 5, y$ is odd and not divisible by 3 , and

1. if $S$ consists of a single $H$-orbit, then for any element $s \in S$,

$$
\left|C_{H}(s)\right| \leq(y+1)|H: \operatorname{Inn} T|
$$

2. if $S$ is the union of two $H$-orbits, then for any element $s \in S$,

$$
\left|C_{H}(s)\right| \leq 2(y+1)|H: \operatorname{Inn} T|
$$

Proof. Suppose that $\Gamma \cong K_{n}$. Since $G$ is edge-transitive, $G$ must be 2-homogeneous on $V(\Gamma)$ (that is, transitive on unordered pairs of vertices). However, all finite 2-hogeneous groups are primitive of affine or almost simple type (see [5, Theorems 4.1B and 9.4B]). Since $G$ is holomorphic simple we conclude that $\Gamma \not K_{n}$.

By Lemma 5.3, the elements of $S$ form either one orbit $B=B^{-1}$ (since $S$ is inverseclosed) of $H$, or two orbits $B, B^{-1}$. Let $k=|S|$. Then if we fix $s \in S$, we have either $S=\{h(s): h \in H\}$ or $S=\{h(s): h \in H\} \cup\left\{h\left(s^{-1}\right): h \in H\right\}$, and $h(s)=s$ if and only if $h \in C_{H}(s)$. Thus for any $s \in S$, we have $k=\left|H: C_{H}(s)\right|$ if $S$ is a single $H$-orbit, or $k=2\left|H: C_{H}(s)\right|$ if $S$ consists of two $H$-orbits.

Now, let $d$ be the number of orbits of $\operatorname{Inn} T$ on $B$. Since $\operatorname{Inn} T$ is normal in $H$, and $H$ is transitive on $B$, it follows that $H$ permutes the Inn $T$-orbits in $B$ transitively. In particular,

$$
d|| H: \operatorname{Inn} T \mid
$$

and all Inn $T$-orbits on $S$ have the same length; let $k^{\prime}$ be that length. Then for any $s \in S$, we have $k^{\prime}=\left|\operatorname{Inn} T: C_{\operatorname{Inn} T}(s)\right|$, and $d k^{\prime}=|B|=\left|H: C_{H}(s)\right|$. 
Standard results on strongly regular graphs include

$$
k(k-\lambda-1)=\mu(|T|-k-1) .
$$

(cf. [9] (10.1), p. 219). This means either

$$
d k^{\prime}(k-\lambda-1)=\mu(|T|-k-1)
$$

or

$$
2 d k^{\prime}(k-\lambda-1)=\mu(|T|-k-1)
$$

(depending on whether $S$ consists of one or two $H$-orbits). As $k^{\prime}|| T \mid$ and $k^{\prime} \mid k$, we have $\operatorname{gcd}\left(k^{\prime},|T|-k-1\right)=1$, so $\operatorname{gcd}(k,|T|-k-1)=\operatorname{gcd}(k,|T|-1)$ must divide both $k / k^{\prime} \in\{d, 2 d\}$, and $|T|-1$. In fact, all nonabelian simple groups have even order, so $\operatorname{gcd}(k,|T|-k-1)$ always divides $d$ and $|T|-1$. Hence, (5B) yields

$$
(|T|-k-1) \mid \operatorname{gcd}(d,|T|-1)(k-\lambda-1) .
$$

In particular, $|T|-k-1 \leq \operatorname{gcd}(d,|T|-1)(k-\lambda-1)$.

Now, $k-\lambda-1 \leq k-1$, so

$$
|T| \leq(\operatorname{gcd}(d,|T|-1)+1) k-\operatorname{gcd}(d,|T|-1)+1 \leq(\operatorname{gcd}(d,|T|-1)+1) k .
$$

Hence,

$$
k \geq|T| /(\operatorname{gcd}(d,|T|-1)+1) .
$$

If $k=\left|H: C_{H}(s)\right|$, that is, if $S$ is an $H$-orbit, we see that

$$
\left|C_{H}(s)\right| \leq|H: \operatorname{Inn} T|(\operatorname{gcd}(d,|T|-1)+1),
$$

and part (1) is proved. If $k=2\left|H: C_{H}(s)\right|$, then similarly we obtain part (2).

Finally, we show that $y \geq 5$ and that $y$ is not divisible by 3 .

In the special case that $y=1$ we have $\operatorname{gcd}(d,|T|-1)=1$ so $(5 \mathrm{C})$ yields $(|T|-k-1) \mid$ $(k-\lambda-1)$. Since $\mu \leq k$ (the number of 2-paths between two vertices cannot be greater than the valency of each vertex), (5B) forces $k=\mu$. But this would mean that each vertex at distance 2 from the vertex $1_{T}$ has exactly the same neighbours as the vertex $1_{T}$. Now, it is not hard to see that the set of all vertices that have exactly the same neighbours as $1_{T}$, forms a block of imprimitivity of $\operatorname{Aut}(\Gamma)$. Therefore, as $G$ is primitive, we get that there are no vertices at distance 2 from the vertex $1_{T}$, in which case $\Gamma$ is complete, which is a contradiction. So $y>1$.

Since $|T|$ is even for every nonabelian simple group, $y$ is odd and in particular $y \neq 2,4$. Suppose that $y=3$. Then $|T|$ is coprime to 3 . The only nonabelian simple groups whose orders are not divisible by 3 are the Suzuki groups. So, $T={ }^{2} B_{2}(q)$ for some $q=2^{2 a+1}$. Now

$$
|T|=q^{2}\left(q^{2}+1\right)(q-1) \equiv 1 \cdot 2 \cdot 1 \equiv 2 \quad(\bmod 3) .
$$

Thus $3 \nmid|T|-1$ for any nonabelian simple group $T$, so $y \neq 3$. Thus $y \geq 5$, and $3 \nmid y$.

In the next result, we quickly dispose of the possibility that the nonabelian simple group $T$ is isomorphic to an alternating or sporadic simple group. The remainder of the paper will be devoted to eliminating the possibility that $T$ is isomorphic to a finite simple group of Lie type. We include the Tits group $\left({ }^{2} F_{4}(2)\right)^{\prime}$ in the list of sporadic groups, as $\left({ }^{2} F_{4}(2)\right)^{\prime}$ is not properly a simple group of Lie type. 
Theorem 5.5. Under Hypotheses 5.2, the group $T$ is not an alternating or a sporadic group.

Proof. For every alternating or sporadic group $T$, we have $\mid$ Out $T \mid \in\{1,2,4\}$. Thus we must have $|H: \operatorname{Inn} T| \in\{1,2,4\}$. The order of every simple group is even, so $y=1$. But this contradicts Lemma 5.4.

Now we begin to deal with finite simple groups of Lie type. Our first step involves presenting a general lower bound on the order of the centralisers in all of these groups.

Remark 5.6. To state the following lemma so as to include the Ree and Suzuki groups, the notation that we use for some of the finite simple groups of Lie type is not the most common. As the notation is not completely standard, we feel at liberty to do this. We denote a simple group of Lie type by ${ }^{r} L_{n}(t)$, where $L=A, \ldots, G$ is the Lie type, $n$ is the rank, $r$ is the order of the graph automorphism of the corresponding Dynkin diagram and $t$ is the size of the field where the group is defined. For instance, ${ }^{2} A_{2}\left(q^{2}\right)$ is the unitary group of order $q^{3}\left(q^{2}-1\right)\left(q^{3}+1\right) / \operatorname{gcd}(3, q+1)$.

Lemma 5.7. For any simple group $T={ }^{r} L_{n}(t)$ of Lie type and any element $x$ of the group $T$, we have $\left|C_{T}(x)\right| \geq\left(t^{1 / r}-1\right)^{n} / d$, where $d$ is the order of the diagonal multiplier (see [4, page xvi, Table 6]).

Proof. Let $G$ be the connected simple algebraic group of rank $n$, of Lie type $L$, of adjoint isogeny type and over the algebraic closure of the field $\mathbb{F}_{t}$ of order $t$. Let $\sigma$ be the LangSteinberg endomorphism of $G$ with fixed point group $G_{\sigma}$ such that $T \subseteq G_{\sigma}$. Let $x$ be an element of $T$. By Lemma 3.4 in [6], we have that $\left|C_{G_{\sigma}}(x)\right| \geq\left(t^{1 / r}-\overline{1}\right)^{n}$. Now, since $T$ has index $d$ in $G_{\sigma}$, we have that $\left|C_{T}(x)\right| \geq\left|C_{G_{\sigma}}(x)\right| / d \geq\left(t^{1 / r}-1\right) / d$. This completes the proof.

Before proving the main result of this section, we prove a lemma about simple groups of Lie type that we will use in our main proof.

Lemma 5.8. Let $T={ }^{r} L_{n}(t)$, where $t=p^{a}$ for some prime $p$, and $\hat{y}=\operatorname{gcd}(\mid$ Out $T|| T \mid-$, 1). If $T$ is not a Ree or a Suzuki group, then $\hat{y} \mid(a / r)$. If T is a Ree or a Suzuki group, then $\hat{y} \mid a$.

Proof. The order of the automorphism group of $T$ can be found in [4] page $\mathrm{xv}$ and is tabulated in Table 5. In particular $\mid$ Out $T \mid=d f g$, where $d$ is the diagonal multiplier, $f$ is the order of the field automorphisms and $g$ is the order of the graph automorphisms of the Dynkin diagram (modulo field automorphisms). By inspection, $d, g$ divide the order of $T$ and $f=a$. Also, by inspection of Table 5 in [4], we have that $r$ divides $|T|$ and $a$ if $T$ is not a Ree or a Suzuki group. Therefore the lemma follows.

Theorem 5.9. Under Hypotheses 5.2, the group $T$ is not a finite simple group of Lie type.

Proof. Assume $T={ }^{r} L_{n}(t)$, where $t=p^{a}$ for some prime $p$. Let $d$ be the diagonal multiplier of $T$. We note that by Lemma 5.4 and Lemma 5.8 (and Hypotheses 5.2), we have that $a / r \geq \operatorname{gcd}(\mid$ Out $T|| T \mid-1) \geq$,5 if $T$ is not a Ree or a Suzuki group and similarly $a \geq \operatorname{gcd}(\mid$ Out $T|| T \mid-1) \geq$,5 if $T$ is a Ree or a Suzuki group.

We use Table 5 of [4] extensively in the rest of the proof. 
Case 1. The simple group $T$ is untwisted and has rank $n$ at least 2.

We first assume that $T$ is not $A_{n}(t)$. From Table 5 in [4], we get $\mid$ Out $T \mid \leq 24 a$. Recall from Hypotheses 5.2 that $y=\operatorname{gcd}(|H: \operatorname{Inn} T|,|T|-1) \leq \mid$ Out $T \mid$. Then, using Lemmas 5.4, 5.7 and 5.8 and the fact that $d \leq 4$ for these groups (see Table 5 in [4]), for $s \in S$ we have

$$
\left(p^{a}-1\right)^{n} / 4 \leq\left|C_{H}(s)\right| \leq 2(24 a)(y+1) \leq 2(24 a)(a+1) .
$$

Since $y \geq 5$ and $3 \nmid y$, this inequality is satisfied only if $n=2$ and $p=2$. But if $n=2, p=2$, then $d=1$, $\mid$ Out $T \mid \leq 6 a$ and the inequality

$$
\left(2^{a}-1\right)^{2} \leq\left|C_{H}(s)\right| \leq 2(6 a)(a+1),
$$

is never satisfied.

If $T=A_{n}(t)$, then we have $d=\operatorname{gcd}\left(n+1, p^{a}-1\right)$ and $\mid$ Out $T \mid=2 d a$. Now, using Lemmas 5.4, 5.7 and 5.8, for $s \in S$ we have

$$
\frac{\left(p^{a}-1\right)^{n}}{\operatorname{gcd}\left(n+1, p^{a}-1\right)} \leq\left|C_{H}(s)\right| \leq 4 \operatorname{gcd}\left(n+1, p^{a}-1\right) a(a+1) .
$$

Since $a \geq 5$, this inequality is never satisfied. (This is straightforward to check by dividing into three cases: $d \leq p^{a}-1$ for $n \geq 4$; $d \leq 4$, for $n=3$; and finally $d \leq 3$ for $n=2$, where if $p=2$ and $a$ is odd we have $d=1$.)

Case 2. The group $T$ is a unitary group.

Set $T={ }^{2} A_{n}(t)$. By Table 5 in [4], we have $d=\operatorname{gcd}\left(n+1, p^{a / 2}+1\right)$ and $\mid$ Out $T \mid=d a$. Now, using Lemmas 5.4, 5.7 and 5.8, for $s \in S$ we have

$$
\frac{\left(p^{a / 2}-1\right)^{n}}{\operatorname{gcd}\left(n+1, p^{a / 2}+1\right)} \leq\left|C_{H}(s)\right| \leq 2 \operatorname{gcd}\left(n+1, p^{a / 2}+1\right) a(a / 2+1) .
$$

Since $a / 2 \geq 5$, this inequality is satisfied only if $n=2, p=2, a=10$. But if $T=$ ${ }^{2} A_{2}\left(2^{10}\right)$, then $|T| \equiv 2(\bmod 5)$, and so $\operatorname{gcd}(|\operatorname{Out} T|,|T|-1)=1<5$, contradicting Lemma 5.4. (The impossibility of satisfying this inequality with other values of $n, p$ and $a$ is straightforward to check by breaking it down into three cases: $d \leq p^{a}+1$ for $n \geq 4$; $d \leq 4$ for $n=3$; and finally $d \leq 3$ for $n=2$.)

Case 3. The simple group $T$ is twisted with $r=2$.

Because of Case 2, we may assume that $T$ is not a unitary group. If $T$ is not a Suzuki or a Ree group (i.e. $T$ is ${ }^{2} D_{n}(t)$ or ${ }^{2} E_{6}(t)$ ), then $d \leq 4$ and $\mid$ Out $T \mid \leq 4 a$. In particular, by Lemmas 5.4, 5.7 and 5.8, we have

$$
\frac{\left(p^{a / 2}-1\right)^{n}}{4} \leq\left|C_{H}(s)\right| \leq 2(4 a)(a / 2+1) .
$$

Since $a / 2 \geq 5$, this inequality is never satisfied.

If $T$ is a Suzuki group or a Ree group (i.e. $T$ is ${ }^{2} B_{2}\left(2^{a}\right),{ }^{2} G_{2}\left(3^{a}\right)$, or ${ }^{2} F_{4}\left(2^{a}\right)$ ), then $d=1, a$ is odd and $\mid$ Out $T \mid=a$. By Lemmas 5.4, 5.7 and 5.8, we have that

$$
\left(p^{a / 2}-1\right)^{n} \leq\left|C_{H}(s)\right| \leq 2 a(a+1) .
$$


Since $a \geq 5$, this inequality is satisfied only if $p=2, n=2$ and $a=5,7$ (i.e. $T=$ $\left.{ }^{2} B_{2}\left(2^{5}\right),{ }^{2} B_{2}\left(2^{7}\right)\right)$. If $T={ }^{2} B_{2}\left(2^{5}\right)$, then $\mid$ Out $T \mid=5$ divides the order of $T$, therefore $\operatorname{gcd}(\mid$ Out $T|| T \mid-1)=,1<5$, contradicting Lemma 5.4. If $T={ }^{2} B_{2}\left(2^{7}\right)$, then $\mid$ Out $T \mid=$ 7 and $|T| \equiv 6 \bmod 7$, therefore $\operatorname{gcd}(|\operatorname{Out} T|,|T|-1)=1<5$, contradicting Lemma 5.4 .

Case 4. The simple group $T$ is the Steinberg triality group ${ }^{3} D_{4}(t)$.

From Table 5 in [4], we have $d=1$ and $\mid$ Out $T \mid=a$. By Lemmas 5.4, 5.7 and 5.8, we have

$$
\left(p^{a / 3}-1\right)^{4} \leq\left|C_{H}(s)\right| \leq 2 a(a / 3+1),
$$

which is never satisfied for $a / 3 \geq 5$.

Case 5. The simple group $T$ is untwisted of rank 1 , and is not a unitary group.

This can only happen if $T$ is the projective special linear group $A_{1}(t)$. The order of the outer automorphism group of $T$ is $a \operatorname{gcd}\left(2, p^{a}-1\right)$, which is $a$ if $p=2$, and $2 a$ otherwise.

Now using Lemmas 5.4 and 5.7, for any $s \in S$, we have

$$
\frac{p^{a}-1}{d} \leq\left|C_{H}(s)\right| \leq \begin{cases}2 a(a+1) & \text { if } p \text { is even } \\ 4 a(a+1) & \text { if } p \text { is odd }\end{cases}
$$

where $d=1$ if $p$ is even, and $d=2$ if $p$ is odd. Now $a \geq 5$, so this inequality is only satisfied when $p^{a}=2^{5}$.

If $T=A_{1}(32)=\operatorname{PSL}(2,32)$, we have $\mid$ Out $T \mid=5$, so $|H: \operatorname{Inn} T| \in\{1,5\}$. By Lemma 5.4, $|H: \operatorname{Inn} T| \geq 5$. Therefore $|H: \operatorname{Inn} T|=5$ and $H=\operatorname{P\Gamma L}(2,32)$. Again by Lemma 5.4, $\left|C_{H}(s)\right| \leq 30$ if $S$ consists of a single $H$-orbit, and $\left|C_{H}(s)\right| \leq 60$ if $S$ is the union of two $H$-orbits. Now, Lemma 5.7 gives $\left|C_{H}(s)\right| \geq 31$, so $S$ must be the union of two $H$-orbits, and by Lemma 5.3, the orbits must have the form $B$ and $B^{-1}$. There are 6 conjugacy classes of elements of $\operatorname{PSL}(2,32)$ in $\operatorname{P\Gamma L}(2,32)$ for which the centralisers of the elements have order at most 60 . First, there is a single conjugacy class of elements of order 11; since there is only one such class, it cannot be in $S$. Next, there are three conjugacy classes of elements of order 31 ; each of these is the conjugacy class represented by a matrix of the form

$$
\left[\begin{array}{cc}
x & 0 \\
0 & x^{-1}
\end{array}\right]
$$

which is conjugate in $\operatorname{PSL}(2,32)$ to its own inverse, since they have the same eigenvalues. Therefore, these three conjugacy classes are inverse-closed, so they cannot be in $S$. Finally, there are two conjugacy classes of elements of order 33. The normaliser of a cyclic group of order 33 is a dihedral group of order 66, where the involution inverts the element in the cycle, so these conjugacy classes are inverse-closed, and again cannot be in $S$. Thus, $T \neq \operatorname{PSL}(2,32)$.

We summarise the results of this section in the following corollary.

Corollary 5.10. Let $\Gamma$ be a connected ve-srg, with $G \leq \operatorname{Aut}(\Gamma)$ acting transitively on the vertices and the edges. Then $G$ cannot be a holomorphic simple group.

Proof. This is an immediate consequence of Theorems 5.5 and 5.9, together with the Classification of Finite Simple Groups. 


\section{References}

[1] K. T. Arasu, D. Jungnickel, S. L. Ma and A. Pott, Strongly regular Cayley graphs with $\lambda-\mu=$ 1, J. Combin. Theory Ser. A 67 (1994), 116-125.

[2] W. G. Bridges and R. A. Mena, Rational circulants with rational spectra and cyclic strongly regular graphs, Ars Combin. 8 (1979), 143-161.

[3] A. E. Brouwer, A. V. Ivanov and M. H. Klin, Some new strongly regular graphs, Combinatorica 9 (1989), 339-344.

[4] J. H. Conway, R. T. Curtis, S. P. Norton, R. A. Parker and R. A. Wilson, Atlas of Finite groups, Clarendon Press, Oxford, 1985.

[5] J. D. Dixon and B. Mortimer, Permutation groups, Graduate texts in Mathematics 163, Springer-Verlag, New York, 1996.

[6] J. Fulman and R. Guralnick, Derangements in simple and primitive groups. Groups, combinatorics \& geometry (Durham, 2001), World Sci. Publ., River Edge, NJ (2003), 99-121.

[7] M. Giudici, C. Li and C. Praeger, Characterizing finite locally $s$-arc transitive graphs with a star normal quotient, J. Group Theory 9 (2006), 641-658.

[8] M. Giudici, C. Li and C. Praeger, Locally $s$-arc transitive graphs with two different quasiprimitive actions, J. Algebra 299 (2006), 863-890.

[9] C. Godsil and G. Royle, Algebraic Graph Theory, Graduate texts in Mathematics 207, SpringerVerlag, New York, 2001.

[10] M. D. Hestenes and D. G. Higman, Rank 3 groups and strongly regular graphs, SIAM-AMS Proc., Vol. IV, Amer. Math. Soc., Providence, R.I. (1971), 141-159.

[11] D. G. Higman, Finite permutation groups of rank 3, Math. Z. 86 (1964), 145-156.

[12] D. G. Higman, Intersection matrices for finite permutation groups, J. Algebra 6 (1967), 22-42.

[13] D. G. Higman, Coherent configurations, I. Rend. Sem. Mat. Univ. Padova 44 (1970), 1-25.

[14] Y. I. Leifman and M. E. Muzychuk, Strongly regular Cayley graphs over the group $\mathbb{Z}_{p^{n}} \oplus \mathbb{Z}_{p^{n}}$, Discrete Math. 305 (2005), 219-239.

[15] A. Malnič, D. Marušič and P. Šparl, On strongly regular bicirculants, European J. Combin. 28 (2007), 891-900.

[16] D. Marušič, Strong regularity and circulant graphs, Discrete Math. 78 (1989), 119-125.

[17] A. Neumaier, Cliques and claws in edge-transitive strongly regular graphs, Math. Z. 174 (1980), 197-202.

[18] C. E. Praeger, Imprimitive symmetric graphs, Ars Combin. 19 A (1985), 149-163.

[19] C. E. Praeger, An O'Nan-Scott Theorem for Finite Quasiprimitive Permutation Groups and an Application to 2-Arc Transitive Graphs, J. London Math. Soc. 47 (1993), 227-239.

[20] C. E. Praeger, Finite normal edge-transitive Cayley graphs, Bull. Austral. Math. Soc. 60 (1999), 207-220.

[21] C. Praeger, J. Saxl and K. Yokoyama, Distance transitive graphs and finite simple groups, Proc. London Math. Soc. (3) 55 (1987), 1-21. 